\title{
Precipitate in Device or Device Ingredient
}

National Cancer Institute

\section{Source}

National Cancer Institute. Precipitate in Device or Device Ingredient. NCI Thesaurus.

Code C62866.

Problem associated with the separation of solid particles from a liquid as the result of a chemical or physical change. 